\title{
AN ALGORITHM FOR COMPUTING A SEQUENCE OF RICHELOT ISOGENIES
}

\author{
Katsuyuki Takashima and Reo Yoshida
}

\begin{abstract}
We show that computation of a sequence of Richelot isogenies from specified supersingular Jacobians of genus- 2 curves over $\mathbb{F}_{p}$ can be executed in $\mathbb{F}_{p^{2}}$ or $\mathbb{F}_{p^{4}}$. Based on this, we describe a practical algorithm for computing a Richelot isogeny sequence.
\end{abstract}

\section{Introduction}

Computing an isogeny between elliptic curves is used in some applications as a new basic cryptographic operation. One example of such an application was proposed in [5] in which a cryptographic hash function from expander graphs consists of computing an sequence of isogenies (see [6] as well). Moreover, there was an attempt to construct a new type of public key cryptosystem using such an operation (see [11]).

We proposed two simple algorithms for practically computing a sequence of 2 -isogenies between supersingular elliptic curves [16]. These algorithms include several square root computations, then they might cause computation in a huge extension field. However, we [16] showed that, if the sequence starts at an appropriate elliptic curve (over $\mathbb{F}_{p^{2}}$ ), then all the computations of the sequence are performed in $\mathbb{F}_{p^{2}}$. This result implies that such computation is practical.

A Richelot isogeny is a natural generalization of 2-isogenies between elliptic curves to that in the genus- 2 case (see $[1,2,3,12]$ etc). Then, we investigate and establish analogous results for a sequence of Richelot isogenies between supersingular Jacobian varieties of dimension 2 .

Section 2 gives a summary of the results in the genus-1 case given in [16]. Section 3 explains the computation for a Richelot isogeny sequence. Section 4 gives a theoretical basis for the proposed algorithms. Section 5 proposes actually an algorithm for computing a sequence of Richelot isogenies.

Received December 15, 2008.

2000 Mathematics Subject Classification. 14Q05, 11Y16, 11T71, 14K02.

Key words and phrases. hyperelliptic curve, genus two, Richelot isogeny, isogeny graph, supersingular curve. 


\section{Previous result: Genus 1 case}

Charles et al. [5] proposed an algorithm for computing a sequence of 2isogenies between supersingular elliptic curves based on Vélu's formulas [14]. In [16], we described simple algorithms based on compact expressions of 2isogenies, without some redundancy in the description in [5].

Let $p$ be an odd prime $>3, \mathbb{F}_{p}$ the finite field of order $p$, and $\overline{\mathbb{F}}_{p}$ an algebraic closure of $\mathbb{F}_{p}$. For $0 \leq i \leq n$, let $E_{i} / \overline{\mathbb{F}}_{p}$ be a supersingular elliptic curve given by the short Weierstrass normal form $Y^{2}=f_{i}(X)$ with $\operatorname{deg}\left(f_{i}\right)=3$. Let $\left(a_{i, 0}, 0\right),\left(a_{i, 1}, 0\right)$, and $\left(a_{i, 2}, 0\right)$ be 2 -torsion points on $E_{i}$. In [16], we considered the computation of the sequence of 2 -isogenies $\phi_{i}$ associated to $\left(a_{i, 0}, 0\right)$ without backtracking:

$$
E_{0} \stackrel{\phi_{0}}{\longrightarrow} E_{1} \stackrel{\phi_{1}}{\longrightarrow} \cdots \stackrel{\phi_{n-2}}{\longrightarrow} E_{n-1} \stackrel{\phi_{n-1}}{\longrightarrow} E_{n}
$$

Here, we denote $\left(a_{i, 1}, 0\right)$ as the 2 -torsion point associated with the backtracking, i.e., the dual isogeny $\hat{\phi}_{i-1}$. Then, we obtained the following simple recurrence formulas between $\left(a_{i, 0}, a_{i, 1}, a_{i, 2}\right)$ and $\left(a_{i+1,0}, a_{i+1,1}, a_{i+1,2}\right)$ :

$$
\begin{aligned}
a_{i+1,1} & =-2 a_{i, 0} \quad \text { and } \\
a_{i+1,0}, a_{i+1,2} & =a_{i, 0} \pm 2\left[\left(a_{i, 0}-a_{i, 1}\right)\left(a_{i, 0}-a_{i, 2}\right)\right]^{\frac{1}{2}} .
\end{aligned}
$$

Here, note that there is a square root term in the RHS of the second formula of $(2)$.

Based on (2), we proposed two simple algorithms for a sequence (1). Moreover, we showed that when appropriately choosing a starting supersingular elliptic curve $E_{0} / \mathbb{F}_{p^{2}}$, all 2 -torsions on $E_{i}$, i.e., $\left(a_{i, m}, 0\right)$, are defined in $\mathbb{F}_{p^{2}}$, and then all the computation of the proposed algorithms stays in $\mathbb{F}_{p^{2}}$.

\section{Preliminaries}

We give several basic facts and fix notations.

\subsection{Hyperelliptic curves of genus 2 and their Jacobians}

Let $p$ be an odd prime $>5$. Then, a hyperelliptic curve of genus 2 over $\overline{\mathbb{F}}_{p}$ is given by

$$
C: Y^{2}=f(X),
$$

where $\operatorname{deg}(f(X))=5$ or 6 and $f(X)$ has no multiple zeros. Let the zeros of $f(X)$ be $\left(a_{0}, \ldots, a_{4}\right)$, or $\left(a_{0}, \ldots, a_{5}\right)$. Then, $P_{m}:=\left(a_{m}, 0\right)$ for $0 \leq m \leq 4$ or 5 are called Weierstrass points (When $\operatorname{deg}(f)=5$, the infinity point gives another Weierstrass point). Given a hyperelliptic curve $C$ of genus 2, we can define a group variety $J_{C}$, the Jacobian. A point $D$ on $J_{C}$ is given by a divisor class of $C$ of degree 0 , which is a formal sum of points on $C$ modulo linear equivalence. When $\operatorname{deg}(f(X))=5, D$ is represented by a pair of polynomials, 
in other words, as a set,

$$
\begin{gathered}
J_{C}=J_{C}\left(\overline{\mathbb{F}}_{p}\right)=\left\{(u(X), v(X)) \in \overline{\mathbb{F}}_{p}[X]^{2}|u(X)| v(X)^{2}-f(X), u(X):\right. \text { monic } \\
\operatorname{deg}(v(X))<\operatorname{deg}(u(X)) \leq 2\},
\end{gathered}
$$

where $\overline{\mathbb{F}}_{p}[X]$ is the polynomial ring whose coefficient field is $\overline{\mathbb{F}}_{p}$. When $\operatorname{deg}(f(X))$ $=6$, a point in $J_{C}$ is given by a pair $(u(X), v(X))$ s.t. $u(X) \mid v(X)^{2}-f(X)$, $u(X)$ : monic, and $\operatorname{deg}(v(X))<\operatorname{deg}(u(X)) \leq 2$, and a (distance) parameter $m \in \mathbb{Z}$, where $0 \leq m \leq 2-\operatorname{deg}(v(X))$. Such a representation is called Mumford representation. An addition of divisors naturally gives an algebraic addition law on $J_{C}$. For details, see $[9,10]$. Jacobian $J_{C}$ is called supersingular if it is isogenous (over $\overline{\mathbb{F}}_{p}$ ) to a product of two supersingular elliptic curves, and a curve $C$ is called supersingular if $J_{C}$ is supersingular.

\subsection{Richelot isogeny}

We explain an isogeny of a hyperelliptic curve of genus 2, called Richelot isogeny $[1,2,3,12]$ etc. First, we specify the notations hereafter.

Let $G_{j}(X) \in \overline{\mathbb{F}}_{p}[X]$ for $j=0,1,2$ be 3 monic polynomials of $\operatorname{deg}\left(G_{j}\right) \leq 2$ such that $\prod_{j=0}^{2} G_{j}(X)$ is of degree 5 or 6 and squarefree. Then

$$
C: Y^{2}=f(X)=d \prod_{j=0}^{2} G_{j}(X)
$$

where $d \in \overline{\mathbb{F}}_{p}^{*}$ is a curve of genus 2. By using coefficients $g_{j, k}$ of $G_{j}(X)=$ $\sum_{k=0}^{2} g_{j, k} X^{k}$, let $M$ be the matrix $\left(g_{j, k}\right)_{0 \leq j, k \leq 2}$. Here, note that if $\operatorname{deg}\left(G_{j}\right)=1$, then $g_{j, 2}=0$. If $\operatorname{deg}\left(G_{j}\right)=2$, we denote the zeros of $G_{j}(X)$ by $a_{2 j}$ and $a_{2 j+1}$, i.e., $G_{j}(X)=\left(X-a_{2 j}\right)\left(X-a_{2 j+1}\right)$. Hereafter, we consider permutations of $\left(a_{0}, \ldots, a_{5}\right)$ for the description of the Richelot isogeny. For that purpose, we use a special symbol " $\infty$ " to treat the case that $G_{j}(X)$ is linear, i.e., $G_{j}(X)=X-a$, where $a=a_{2 j}$ or $a_{2 j+1}$. Then, we consider that $a$ and $\infty$ are the two zeros of $G_{j}(X)$, and treat permutations of 6 elements $\left(a_{0}, \ldots, a_{5}\right)$ including $\infty$.

Suppose that the determinant of $M=\left(g_{j, k}\right)_{0 \leq j, k \leq 2}$ is non-zero. Hereafter, prime "" means differentiation by the variable $X$. We then define the bracket product $\left[G_{j+1}(X), G_{j+2}(X)\right]$ and its transform to the monic one, $\tilde{G}_{j}(X)$, below.

$$
\begin{aligned}
{\left[G_{j+1}(X), G_{j+2}(X)\right] } & :=G_{j+1}^{\prime}(X) G_{j+2}(X)-G_{j+2}^{\prime}(X) G_{j+1}(X), \\
\tilde{G}_{j}(X) & :=c_{j}^{-1}\left[G_{j+1}(X), G_{j+2}(X)\right]
\end{aligned}
$$

where $c_{j}$ is the leading coefficient of $\left[G_{j+1}(X), G_{j+2}(X)\right]$. Here, and in similar places throughout this paper, we will take addition with respect to the index of $G$ to mean addition modulo 3 . Then, the degree of $\prod_{j=0}^{2} \tilde{G}_{j}(X)$ is 5 or 6 [12]. Let $\tilde{f}(X):=\tilde{d} \prod_{j=0}^{2} \tilde{G}_{j}(X)$, where $\tilde{d}:=d \cdot c_{0} c_{1} c_{2} \cdot \operatorname{det}(M)^{-1}$. Using $\tilde{f}(X)$, we 
then obtain a curve of genus 2

$$
\tilde{C}: Y^{2}=\tilde{f}(X)=\tilde{d} \prod_{j=0}^{2} \tilde{G}_{j}(X) \text { with } \tilde{d}:=d \cdot c_{0} c_{1} c_{2} \cdot \operatorname{det}(M)^{-1} .
$$

The curve $\tilde{C}$ is called a Richelot dual of $C$. Here, we call the above correspondence Richelot operator $\mathcal{R}$ according to B. Smith [12].

$$
\mathcal{R}:\left(G_{0}(X), G_{1}(X), G_{2}(X), d\right) \mapsto\left(\tilde{G}_{0}(X), \tilde{G}_{1}(X), \tilde{G}_{2}(X), \tilde{d}\right) .
$$

However, this $\mathcal{R}$ is slightly different from that in [12].

Associated with a Weierstrass point $P_{0}=\left(a_{0}, 0\right)$, the Richelot isogeny is given by

$$
\begin{aligned}
\phi: J_{C} & \rightarrow J_{\tilde{C}} \\
D=\left[(x, y)-P_{0}\right] & \mapsto \phi(D)=\left[\left(z_{1}, t_{1}\right)-\left(z_{2},-t_{2}\right)\right],
\end{aligned}
$$

where $[\cdot]$ means linear equivalence class, $z_{1}$ and $z_{2}$ are the zeros with respect to $z$ of

$$
U_{x}(z)=\sum_{k=0}^{2} U_{x, k} z^{k}:=G_{1}(x) \tilde{G}_{1}(z)+G_{2}(x) \tilde{G}_{2}(z),
$$

and $t_{\ell}$ satisfies

$$
y t_{\ell}=\sum_{k=0}^{2} V_{x, k} z_{\ell}^{k}
$$

where $\sum_{k=0}^{2} V_{x, k} z_{\ell}^{k}:=d G_{1}(x) \tilde{G}_{1}\left(z_{\ell}\right)\left(x-z_{\ell}\right)$ for $\ell=1,2$. We note that $\left(z_{1}, t_{1}\right)$ and $\left(z_{2}, t_{2}\right)$ are points on $\tilde{C}$. The kernel of $\phi$ is explicitly given by the Weierstrass points, and it is isomorphic to $(\mathbb{Z} / 2 \mathbb{Z})^{2}$. For details, see $[1,12]$.

\subsection{A sequence of Richelot isogenies}

Let $C$ be given by (3). Richelot isogenies from $J_{C}$ are determined by splitting $\left(G_{0}(X), G_{1}(X), G_{2}(X)\right)$ of $f(X)$. This corresponds to a splitting of the zeropoints of $f(X)$ into three pairs, i.e., $\left(a_{0}, a_{1}\right),\left(a_{2}, a_{3}\right)$, and $\left(a_{4}, a_{5}\right)$. Therefore, the number of Richelot isogenies from $C$ is $\left(\begin{array}{l}6 \\ 2\end{array}\right) \cdot\left(\begin{array}{l}4 \\ 2\end{array}\right) / 3 !=15$. We consider computing a walk consisting of Richelot isogenies

$$
J_{0} \stackrel{\phi_{0}}{\longrightarrow} J_{1} \stackrel{\phi_{1}}{\longrightarrow} \cdots \stackrel{\phi_{n-2}}{\longrightarrow} J_{n-1} \stackrel{\phi_{n-1}}{\longrightarrow} J_{n}
$$

without backtracking, i.e., $\phi_{i+1}$ is not the dual of $\phi_{i}$ for $i=0, \ldots, n-2$. Hence, at each step, there exist $14=15-1$ possible choices to go forward. In (7), $J_{i}$ is the Jacobian of $C_{i}$, which is given below.

$$
C_{i} / \overline{\mathbb{F}}_{p}: Y^{2}=f_{i}(X)=d_{i} \prod_{j=0}^{2} G_{i, j}(X),
$$


where

$$
G_{i, j}(X)=\sum_{k=0}^{2} g_{i, j, k} X^{k}= \begin{cases}\left(X-a_{i, 2 j}\right)\left(X-a_{i, 2 j+1}\right) & \text { if } \operatorname{deg}\left(G_{i, j}\right)=2 \\ X-a_{i, 2 j} \text { or } X-a_{i, 2 j+1} & \text { if } \operatorname{deg}\left(G_{i, j}\right)=1\end{cases}
$$

where $d_{i} \neq 0$ and $\operatorname{det}\left(M_{i}\right) \neq 0$ for $M_{i}:=\left(g_{i, j, k}\right)_{0 \leq j, k \leq 2}$. For the Richelot dual $\tilde{C}_{i+1}$ (after applying $\phi_{i}$ to $J_{C_{i}}$ ), we use similar notation $\tilde{G}_{i+1, j}(X), \tilde{a}_{i+1, m}$, and $\tilde{d}_{i+1}$ for the corresponding ones, respectively.

Here, we note that, if $\operatorname{det}\left(M_{i}\right)=0$, then $J_{C_{i}}$ has an isogeny to a product of elliptic curves $E_{1} \times E_{2}$ [12]. We do not consider such special cases in the presentation of sequence computation hereafter.

From the above, we have 14 possibilities to proceed to the next Jacobian at $i \geq 1$. When $i=0$, we choose 14 possibilities from $J_{0}$ at the beginning. We then associate a walk data $\omega=b_{0} b_{1} \cdots b_{n-1} \in \mathcal{W}=\{0, \ldots, 13\}^{n}$ with a walk (7). Then, the correspondence is bijective as indicated below.

$$
\mathcal{W}=\{0, \ldots, 13\}^{n} \longleftrightarrow\left\{\begin{array}{l}
\text { a sequence }(7) \text { of Richelot isogenies } \phi_{i} \\
\text { starting from } J_{0} \text { without backtracking }
\end{array}\right\},
$$

where (7) starts from one of the candidate Jacobians chosen as above. The goal is to compute the $C_{n}$ from $C_{0}$ and a walk data $\omega \in \mathcal{W}=\{0, \ldots, 13\}^{n}$.

For $i=1, \ldots, n-1$, the $i$-th step in (7) for computing $\phi_{i}$ consists of the following 2 procedures

1: Permutation of the zero-points of $f_{i}(X)$,

2: Isogeny calculation by the Richelot operator, i.e.,

$$
\tilde{G}_{i+1, j}(X)=c_{i, j}^{-1}\left[G_{i, j+1}(X), G_{i, j+2}(X)\right],
$$

where $c_{i, j}$ is the leading coefficient of $\left[G_{i, j+1}(X), G_{i, j+2}(X)\right]$.

The flow of the computation is given below.

$$
\begin{aligned}
& \text { : } \\
& \overbrace{\tilde{a}_{i, 0}, \tilde{a}_{i, 1}}^{\tilde{G}_{i, 0}(X)} \overbrace{\tilde{a}_{i, 2}, \tilde{a}_{i, 3},}^{\tilde{G}_{i, 1}(X)} \overbrace{\tilde{a}_{i, 4}, \tilde{a}_{i, 5}}^{\tilde{G}_{i, 2}(X)} \\
& \Downarrow \\
& \overbrace{a_{i, 0}, a_{i, 1}}^{G_{i, 0}(X)}, \overbrace{a_{i, 2}, a_{i, 3}}^{G_{i, 1}(X)}, \overbrace{a_{i, 4}, a_{i, 5}}^{G_{i, 2}(X)} \\
& \Downarrow \\
& \overbrace{\tilde{a}_{i+1,0}, \tilde{a}_{i+1,1}}^{\tilde{G}_{i+1,0}(X)}, \overbrace{\tilde{a}_{i+1,2}, \tilde{a}_{i+1,3}}^{\tilde{G}_{i+1,1}(X)}, \overbrace{\tilde{a}_{i+1,4}, \tilde{a}_{i+1,5}}^{\tilde{G}_{i+1,2}(X)} \\
& \Downarrow \\
& \text { Richelot op. } \\
& \text { Perm. } \\
& \text { Richelot op. } \\
& \text { Perm. }
\end{aligned}
$$


Here, one of $\left\{\tilde{a}_{i, m}\right\}$ and one of $\left\{a_{i, m}\right\}$ are $\infty$ when $\operatorname{deg}\left(\tilde{f}_{i}\right)=5\left(=\operatorname{deg}\left(f_{i}\right)\right)$. Similarly, one of $\left\{\tilde{a}_{i+1, m}\right\}$ is $\infty$ when $\operatorname{deg}\left(\tilde{f}_{i+1}\right)=5\left(=\operatorname{deg}\left(f_{i+1}\right)\right)$. We give an explicit expression of $\tilde{a}_{i+1, m}$ by $a_{i, 0}, \ldots, a_{i, 5}$ in Section 5.2 .

To permute 6 zero-points of $\tilde{G}_{i, 0}(X), \tilde{G}_{i, 1}(X)$ and $\tilde{G}_{i, 2}(X)$, we must solve the quadratic equations $\tilde{G}_{i, j}(X)=0$ for $j=0,1,2$. Hence, square root computations are the most time-consuming as in the genus 1 case. See Section 2 and $[16]$.

\section{Defining field of Weierstrass points}

Since we take square roots at each step in (7), in the worst case, one might end up doing arithmetic in a prohibitively huge finite field, e.g., $\mathbb{F}_{p^{2^{n}}}$, even if we start at a curve over $\mathbb{F}_{p}$. However, here we show that if we choose a starting point appropriately, then all the computations for (7) stay in $\mathbb{F}_{p^{2}}$ or $\mathbb{F}_{p^{4}}$. Actually, we prove such computations are performed in $\mathbb{F}_{p^{2}}$ or $\mathbb{F}_{p^{4}}$ by starting a sequence at the following two types of hyperelliptic curves.

$$
\begin{aligned}
& (\text { Type } I) C^{I} / \mathbb{F}_{p}: Y^{2}=X^{5}+\alpha \text { for } p \equiv 4 \bmod 5 \\
& (\text { Type } I I) C^{I I} / \mathbb{F}_{p}: Y^{2}=X^{5}+\alpha \text { for } p \equiv 2,3 \bmod 5 .
\end{aligned}
$$

Hereafter, we denote $r$ to be 2 for Type I curves and 4 for Type II curves. We let $q$ be $p^{r}$. Using this notation, Theorem 4.1 shows that all computations stay in $\mathbb{F}_{q}$ when we start at $J_{C^{I}}$ or $J_{C^{I I}}$.

\subsection{The main theorem}

Theorem 4.1. If a sequence of Richelot isogenies (7) starts at $J_{C^{I}}$ or $J_{C^{I I}}$, then the following holds for all $i$ :

$$
J_{i}\left(\mathbb{F}_{q}\right) \cong\left(\mathbb{Z} /\left(q^{\frac{1}{2}}+1\right) \mathbb{Z}\right)^{4} .
$$

In particular, all Weierstrass points on $C_{i}$ are defined over $\mathbb{F}_{q}$ and all the computations of the sequence $(7)$ are performed in $\mathbb{F}_{q}$.

Proof. First, note that if $(10)$ holds for all $i$, then $J_{i}\left(\mathbb{F}_{q}\right)[2] \cong(\mathbb{Z} / 2 \mathbb{Z})^{4}$ because $p$ is an odd prime. From Lemma 4.2, then all the computation of $(7)$ are performed in $\mathbb{F}_{q}$.

Therefore, we must show the group structure (10) of $J_{i}\left(\mathbb{F}_{q}\right)$. We show this by induction. The following Lemma 4.3 shows that (10) holds at the starting point $J_{0}=J_{C^{I}}$ or $J_{C^{I I}}$. In addition, Lemma 4.4 shows that (10) holds for all $i$ inductively.

Lemma 4.2. If $J_{i}\left(\mathbb{F}_{q}\right)[2] \cong(\mathbb{Z} / 2 \mathbb{Z})^{4}$, the Richelot isogeny $\phi_{i}$ in $(7)$ is defined over $\mathbb{F}_{q}$.

Lemma 4.3. For the curves $C^{I}$ and $C^{I I}$,

$$
\begin{aligned}
J_{C^{I}}\left(\mathbb{F}_{p^{2}}\right) \cong(\mathbb{Z} /(p+1) \mathbb{Z})^{4} \text { and } \\
J_{C^{I I}}\left(\mathbb{F}_{p^{4}}\right) \cong\left(\mathbb{Z} /\left(p^{2}+1\right) \mathbb{Z}\right)^{4} .
\end{aligned}
$$


Lemma 4.4. If (10) holds for $J_{i}$ in $(7)$, then $J_{i}\left(\mathbb{F}_{q}\right) \cong J_{i+1}\left(\mathbb{F}_{q}\right)$ as a group.

We next prove Lemmas 4.2, 4.3 and 4.4.

\subsection{Proofs of Lemmas $4.2,4.3$ and 4.4}

Proof of Lemma 4.2. All Weierstrass points $\left(a_{i, m}, 0\right)$ of $C_{i}$, where $m=0, \ldots, 5$, are defined in $\mathbb{F}_{q}$ from the assumption of Lemma 4.2. Therefore, all the coefficients of $G_{i, j}(X)$ and $\tilde{G}_{i+1, j}(X)$, which are defined in Section 3.3, are in $\mathbb{F}_{q}$. Therefore, all coefficients $U_{x, k}$ in (6) and $V_{x, k}$ in (6) for $k=0,1,2$, are in $\mathbb{F}_{q}[x]$.

Because $z_{1}$ and $z_{2}$ are two zeros of $U_{x}$ in (6), the $u$-polynomial of the Mumford representation of $\phi(D)$ in (5) is equal to $U_{x}$ up to a constant multiple, and it is defined over $\mathbb{F}_{q}$. Let $V(z):=\sum_{k=0}^{2}\left(V_{x, k} / y\right) z^{k}$. Then, from (6), $t_{\ell}=V\left(z_{\ell}\right) \in \mathbb{F}_{q}(y)[x]$ for $\ell=1,2$. The $v$-polynomial of the Mumford representation of $\phi(D)$ in (5) is given by the remainder of $V(z)$ by the $u$-polynomial, which is defined over $\mathbb{F}_{q}$. Hence, all the coefficients of the $v$-polynomial are also in $\mathbb{F}_{q}(x, y)$.

This means that the isogeny $\phi_{i}$ is defined over $\mathbb{F}_{q}$.

We denote the characteristic polynomial of the $p^{r}$-th power Frobenius on a Jacobian $J / \mathbb{F}_{p}$ by $h_{r}(T)$. Let the characteristic polynomial $h_{1}(T)$ be given by $h_{1}(T)=\prod_{\ell=1}^{2}\left(T-\pi_{\ell}\right)\left(T-\bar{\pi}_{\ell}\right)$.

Lemma 4.3 follows from the following Facts 4.5, 4.6 and 4.7. Fact 4.5 gives $h_{1}(T)$ for $J_{C^{I}} / \mathbb{F}_{p}$ and $J_{C^{I I}} / \mathbb{F}_{p}$. Fact 4.6 gives a fundamental relation between $h_{1}(T)$ and $h_{r}(T)$. Fact 4.7 determines the group structure of $\mathbb{F}_{q}$-rational points of Jacobians from the characteristic polynomials $h_{r}(T)$.

Proof of Lemma 4.3. We first show (11) for $J_{C^{I}}$. Without loss of generality, we let $\pi_{1}=\pi_{2}=\sqrt{-p} \in \mathbb{C}$ from (13) in Fact 4.5. Then, all $\pi_{1}^{2}=\pi_{2}^{2}=\bar{\pi}_{1}^{2}=$ $\bar{\pi}_{2}^{2}=-p$, i.e., $h_{2}(T)=(T+p)^{4}$ from Fact 4.6. Fact 4.7 shows that the group structure is $J_{C^{I}}\left(\mathbb{F}_{p^{2}}\right) \cong(\mathbb{Z} /(p+1) \mathbb{Z})^{4}$.

We next show (12) for $J_{C^{I I}}$. Without loss of generality, we let $\pi_{1}=\zeta_{8} \sqrt{p}$ and $\pi_{2}=\zeta_{8}^{3} \sqrt{p} \in \mathbb{C}$, where $\zeta_{8}$ is a primitive 8 -th root of unity, from (14) in Fact 4.5. Then, all $\pi_{1}^{4}=\pi_{2}^{4}=\bar{\pi}_{1}^{4}=\bar{\pi}_{2}^{4}=-p^{2}$, i.e., $h_{4}(T)=\left(T+p^{2}\right)^{4}$ from Fact 4.6 , and $J_{C^{I I}}\left(\mathbb{F}_{p^{4}}\right) \cong\left(\mathbb{Z} /\left(p^{2}+1\right) \mathbb{Z}\right)^{4}$ from Fact 4.7 .

Fact 4.5 ([8, Prop. 1.13], [7, Example 5.1]). Two curves $C^{I}$ and $C^{I I}$ are supersingular, and $h_{1}(T)$ for each curve is given by

$$
\begin{aligned}
& \text { (I) } h_{1}(T)=\left(T^{2}+p\right)^{2} \text { and } \\
& \text { (II) } h_{1}(T)=T^{4}+p^{2}
\end{aligned}
$$

respectively. 
Fact 4.6 ([4, Ch.14 Theorem 14.17]). Let the characteristic polynomial $h_{1}(T)$ be given by the following:

$$
h_{1}(T)=\prod_{\ell=1}^{2}\left(T-\pi_{\ell}\right)\left(T-\bar{\pi}_{\ell}\right),
$$

where $\pi_{\ell}$ in $\mathbb{C}$ for $\ell=1,2$ s.t. $\left|\pi_{\ell}\right|=\sqrt{p}$. Then, the characteristic polynomials $h_{r}(T)$ are given by

$$
h_{r}(T)=\prod_{\ell=1}^{2}\left(T-\pi_{\ell}^{r}\right)\left(T-\bar{\pi}_{\ell}^{r}\right) .
$$

Fact 4.7 ([15, Theorem 2]). Let $q$ be $p^{r}$, A a supersingular abelian surface over $\mathbb{F}_{q}$, and $h_{r}(T)$ the characteristic polynomial of $A / \mathbb{F}_{q}$. Suppose that $h_{r}(T)$ has the decomposition $h_{r}(T)=\prod_{\ell=1}^{\eta} w_{\ell}(T)^{e_{\ell}}$, where $w_{\ell}(T)$ is $\mathbb{Q}$-irreducible for $\ell=1, \ldots, \eta$. Then,

$$
A\left(\mathbb{F}_{q}\right) \cong \bigoplus_{\ell=1}^{\eta}\left(\mathbb{Z} /\left|w_{\ell}(1)\right| \mathbb{Z}\right)^{e_{\ell}}
$$

except for $A$ in the following cases:

(i) $h_{r}(T)=\left(T^{2}-q\right)^{2}$,

(ii) $r$ is odd and $h_{r}(T)=\left(T^{2}+q\right)^{2}$.

Lemma 4.4 follows from Facts 4.7, 4.8 and Lemma 4.2. Fact 4.8 is (a part of) a famous classification theorem given by Tate [13].

Proof of Lemma 4.4. Since $\phi_{i}$ are defined over $\mathbb{F}_{q}$ from Lemma 4.2, the characteristic polynomials of the $q$-th power Frobenius, $h_{r}(T)$, are the same for $J_{i}$ and $J_{i+1}$. Because the polynomial $h_{r}(T)$ is $\left(T+q^{\frac{1}{2}}\right)^{4}$, we conclude that $J_{i+1}\left(\mathbb{F}_{q}\right) \cong J_{i}\left(\mathbb{F}_{q}\right)$ from Fact 4.7 .

Fact 4.8 ([13, Theorem 1 , a part of $(\mathrm{c})])$. Let $A$ and $B$ be abelian varieties over a finite field $\mathbb{F}$, and let $h_{A}$ and $h_{B}$ be characteristic polynomials of their Frobenius endomorphisms relative to $\mathbb{F}$. Then, the following statements equivalent:

(i) $A$ and $B$ are $\mathbb{F}$-isogenous.

(ii) $h_{A}=h_{B}$.

\section{Algorithm for computing a sequence}

In this section, we propose an algorithm for computing a sequence of Richelot isogenies (Algorithms 1 and 2). We give some notations for that. Using the notation (8), let $\xi_{i}$ be a tuple of $6 a_{i, m}$ 's, namely, $\xi_{i}=\left(a_{i, 0}, \ldots, a_{i, 5}\right)$ (possibly including $\infty)$ and let $S_{i}$ be the data consisting of $\xi_{i}$ and the mulplicative factor $d_{i}$, which determines the defining equation of $C_{i}$, that is,

$$
S_{i}:=\left(\xi_{i}, d_{i}\right)=\left(\left(a_{i, 0}, \ldots, a_{i, 5}\right), d_{i}\right) .
$$


We use a similar notation,

$$
\tilde{S}_{i}:=\left(\tilde{\xi}_{i}, \tilde{d}_{i}\right)=\left(\left(\tilde{a}_{i, 0}, \ldots, \tilde{a}_{i, 5}\right), \tilde{d}_{i}\right)
$$

as well. Since the starting curve $C_{0}$ is $C^{I}$ or $C^{I I}$, we let $\tilde{\xi}_{0}$ consist of 5 zeropoints $\tilde{a}_{0}, \ldots, \tilde{a}_{4}$ of $f(X)$ for $C_{0}$ and $\infty$. Then, $\tilde{S}_{0}=\left(\tilde{\xi}_{0}, 1\right)$, and, computing a sequence of Richelot isogenies (7) is represented by computing the following sequence consisting of $S_{i}$ and $\tilde{S}_{i}$.

$$
\begin{aligned}
& \tilde{S}_{0} \stackrel{\stackrel{\text { Perm }}{\longrightarrow}}{\longrightarrow} S_{0} \stackrel{\phi_{0}}{\longrightarrow} \tilde{S}_{1} \stackrel{\text { Perm }_{1}}{\longrightarrow} S_{1} \stackrel{\phi_{1}}{\longrightarrow} \tilde{S}_{2} \stackrel{\text { Perm }}{\longrightarrow} \\
& \cdots \quad \stackrel{\phi_{n-2}}{\longrightarrow} \tilde{S}_{n-1} \stackrel{\text { Perm }}{\longrightarrow} S_{n-1} \stackrel{\phi_{n-1}}{\longrightarrow} \tilde{S}_{n},
\end{aligned}
$$

where $\operatorname{Perm}_{i}$ for $i=0, \ldots, n-1$ are permutations of the Weierstrass points determined by the $i$-th bit $b_{i}$ of a walk data $\omega=b_{0} \cdots b_{n-1}$, and $\phi_{i}$ are Richelot operators. Perm ${ }_{i}$ does not change the multiplicative factor $\tilde{d}_{i}$. Hence, $\tilde{d}_{i}=d_{i}$ for $i=0, \ldots, n-1$, where $d_{i}$ and $\tilde{d}_{i}$ are a component of $S_{i}$ and $\tilde{S}_{i}$ in (15), respectively.

\subsection{Permutation function}

Here, we give function Perm to permute the Weierstrass points. In Section 3.2 , we observed that a splitting $\left(G_{0}(X), G_{1}(X), G_{2}(X)\right)$ of $f(X)$ in $(3)$ leads to a Richelot isogeny $\phi$. Let $\mathfrak{S}_{6}$ be the symmetric group of degree 6 , acting on $\{0, \ldots, 5\}$. Then, the above splitting is given by some permutation $\sigma \in \mathfrak{S}_{6}$ of the zeros $\left\{a_{0}, \ldots, a_{5}\right\}$ of $f$, i.e., then, the splitting is given by $\left(a_{\sigma(0)}, a_{\sigma(1)}\right)$, $\ldots,\left(a_{\sigma(4)}, a_{\sigma(5)}\right)$. Smith [12] showed that the following set $H$ of permutations gives all representatives, which give 14 non-isomorphic Jacobians. See Table 9.1 in $[12]$.

$$
\begin{aligned}
H:=\{ & (0,1,3,5,2,4),(1,3,5,2,4),(0,3,1,5,4,2),(0,5,1),(0,2)(1,5,4,3), \\
& (0,5),(0,1,2,3,4,5),(1,2,3,4,5),(0,2,5,3)(1,4),(0,2,5,3,1,4), \\
& (0,4,3,2,1),(0,3,2)(1,5,4),(0,4,2,1),(0,4,2)\} .
\end{aligned}
$$

Hereafter, the elements in $H$ are ordered as above.

Perm takes as input an ordered tuple $\left(a_{0}, \ldots, a_{5}\right)$ and $b \in\{0, \ldots, 13\}$, and outputs the ordered tuple $\left(a_{\sigma(0)}, \ldots, a_{\sigma(5)}\right)$, where $\sigma \in \mathfrak{S}_{6}$ is the $b$-th element in $H$. It is easy to see that the identity permutation gives the backtracking isogeny.

\subsection{Richelot operator computation: Formulas for Weierstrass points}

We give explicit formulas for $\tilde{a}_{2 j}, \tilde{a}_{2 j+1}$, and $\tilde{d}$ obtained by applying the Richelot operator $\mathcal{R}$. The following cases occur according to $\operatorname{deg}\left(G_{i, j+1}\right)$, $\operatorname{deg}\left(G_{i, j+2}\right)$, and $\operatorname{deg}\left(\tilde{G}_{i+1, j}\right)$, where $j=0,1,2$.

From (4), in the Richelot operator computation, $d$ is multiplied by $\prod_{j=0}^{2} c_{j}$ and $\left(\operatorname{det}\left(g_{j, k}\right)\right)^{-1}$. The former factor is from the leading coefficients of the 
brackets $\left[G_{i, j+1}(X), G_{i, j+2}(X)\right]$, then we describe the effect in Section 5.2. We treat the update of $\tilde{d}$ using the latter factor in Section 5.3.

5.2.1. Case that $\operatorname{deg}\left(\boldsymbol{G}_{\boldsymbol{i}, \boldsymbol{j}+\mathbf{1}}\right)=\operatorname{deg}\left(\boldsymbol{G}_{\boldsymbol{i}, \boldsymbol{j}+\mathbf{2}}\right)=\mathbf{2}$. From (9), zeros $\tilde{a}_{2 j}$ and $\tilde{a}_{2 j+1}$ of $\tilde{G}_{i+1, j}(X)$ are related to zeros $a_{2(j+1)}, a_{2(j+1)+1}, a_{2(j+2)}$, and $a_{2(j+2)+1}$ of $G_{i, j+1}(X), G_{i, j+2}(X)$ as follows:

$$
\begin{gathered}
{\left[G_{i, j+1}(X), G_{i, j+2}(X)\right]=G_{i, j+1}^{\prime}(X) G_{i, j+2}(X)-G_{i, j+2}^{\prime}(X) G_{i, j+1}(X)} \\
=\left(a_{2(j+1)}+a_{2(j+1)+1}-a_{2(j+2)}-a_{2(j+2)+1}\right) X^{2} \\
\quad-2\left(a_{2(j+1)} a_{2(j+1)+1}-a_{2(j+2)} a_{2(j+2)+1}\right) X \\
\quad+a_{2(j+1)} a_{2(j+1)+1}\left(a_{2(j+2)}+a_{2(j+2)+1}\right) \\
-a_{2(j+2)} a_{2(j+2)+1}\left(a_{2(j+1)}+a_{2(j+1)+1}\right) .
\end{gathered}
$$

Let $\vartheta_{j}:=a_{2(j+1)}+a_{2(j+1)+1}-a_{2(j+2)}-a_{2(j+2)+1}, \lambda_{j+1}:=a_{2(j+1)} a_{2(j+1)+1}$, and $\lambda_{j+2}:=a_{2(j+2)} a_{2(j+2)+1}$.

Subcase that $\operatorname{deg}\left(\tilde{\boldsymbol{G}}_{\boldsymbol{i}+\mathbf{1 , j}}\right)=\mathbf{2}$. If $\vartheta_{j} \neq 0, \operatorname{deg}\left(\tilde{G}_{i+1, j}\right)=2$ and then $(16)$ is equal to

$$
\vartheta_{j} \tilde{G}_{i+1, j}(X)=\vartheta_{j}\left(X-\tilde{a}_{2 j}\right)\left(X-\tilde{a}_{2 j+1}\right) .
$$

A quarter of the discriminant of the quadratic $\left[G_{i, j+1}(X), G_{i, j+2}(X)\right]$ is

$$
\begin{aligned}
\delta_{j}= & \left(a_{2(j+1)}-a_{2(j+2)}\right)\left(a_{2(j+1)}-a_{2(j+2)+1}\right) \\
& \left(a_{2(j+1)+1}-a_{2(j+2)}\right)\left(a_{2(j+1)+1}-a_{2(j+2)+1}\right) .
\end{aligned}
$$

That is, $\delta_{j}$ is given by the product of the differences between the zero-points of $G_{i, j+1}(X)$, i.e., $a_{2(j+1)}$ and $a_{2(j+1)+1}$, and the zero-points of $G_{i, j+2}(X)$, i.e., $a_{2(j+2)}$ and $a_{2(j+2)+1}$.

Hence, $\tilde{a}_{2 j}$ and $\tilde{a}_{2 j+1}$ are given by

$$
\tilde{a}_{2 j}, \tilde{a}_{2 j+1}=\frac{\lambda_{j+1}-\lambda_{j+2} \pm \delta_{j}^{\frac{1}{2}}}{\vartheta_{j}} .
$$

The multiplicative factor $\tilde{d}$ is updated to $\tilde{d} \cdot \vartheta_{j}$.

Subcase that $\operatorname{deg}\left(\tilde{\boldsymbol{G}}_{\boldsymbol{i}+\mathbf{1}, \boldsymbol{j}}\right)=\mathbf{1}$. If $\vartheta_{j}=0,(16)$ is linear, i.e., $\operatorname{deg}\left(\tilde{G}_{i+1, j}\right)=1$. Then, the root of $\tilde{G}_{i+1, j}(X)=0, \tilde{a}_{2 j}$, is given by

$$
\tilde{a}_{2 j}=\frac{a_{2(j+1)}+a_{2(j+1)+1}}{2}
$$

since $a_{2(j+1)}+a_{2(j+1)+1}=a_{2(j+2)}+a_{2(j+2)+1}$.

The leading coefficient of $\tilde{G}_{i+1, j}(X)$ is $-2\left(\lambda_{j+1}-\lambda_{j+2}\right)$. Then $\tilde{d}$ is updated to $-2\left(\lambda_{j+1}-\lambda_{j+2}\right) \cdot \tilde{d}$. 
5.2.2. Case that $\operatorname{deg}\left(G_{i, j+1}\right)=1$ or $\operatorname{deg}\left(G_{i, j+2}\right)=1$. First, we consider the case that $\operatorname{deg}\left(G_{i, j+1}\right)=1$, i.e., $G_{i, j+1}(X)$ is linear. We obtain formulas for $\tilde{a}_{2 j}, \tilde{a}_{2 j+1}$ as follows: Let $G_{i, j+1}(X)=X-a_{2(j+1)}, G_{i, j+2}(X)=(X-$ $\left.a_{2(j+2)}\right)\left(X-a_{2(j+2)+1}\right)$. Then,

$$
\begin{aligned}
& {\left[G_{i, j+1}(X), G_{i, j+2}(X)\right] } \\
= & -\tilde{G}_{i+1, j}(X) \\
= & -\left[X^{2}-2 a_{2(j+1)} X+\left(a_{2(j+2)}+a_{2(j+2)+1}\right) a_{2(j+1)}-a_{2(j+2)} a_{2(j+2)+1}\right] .
\end{aligned}
$$

Let $\delta_{j}:=\left(a_{2(j+1)}-a_{2(j+2)}\right)\left(a_{2(j+1)}-a_{2(j+2)+1}\right)$. Then, we obtain

$$
\tilde{a}_{2 j}, \tilde{a}_{2 j+1}=a_{2(j+1)} \pm \delta_{j}^{\frac{1}{2}} .
$$

Next, we consider the case that $\operatorname{deg}\left(G_{i, j+2}\right)=1$. Let $G_{i, j+1}(X)=(X-$ $\left.a_{2(j+1)}\right)\left(X-a_{2(j+1)+1}\right), G_{i, j+2}(X)=X-a_{2(j+2)}$. Then,

$$
\begin{aligned}
& {\left[G_{i, j+1}(X), G_{i, j+2}(X)\right] } \\
= & \tilde{G}_{i+1, j}(X) \\
= & X^{2}-2 a_{2(j+2)} X+\left(a_{2(j+1)}+a_{2(j+1)+1}\right) a_{2(j+2)}-a_{2(j+1)} a_{2(j+1)+1} .
\end{aligned}
$$

Let $\delta_{j}:=\left(a_{2(j+2)}-a_{2(j+1)}\right)\left(a_{2(j+2)}-a_{2(j+1)+1}\right)$. Then, we obtain

$$
\tilde{a}_{2 j}, \tilde{a}_{2 j+1}=a_{2(j+2)} \pm \delta_{j}^{\frac{1}{2}} .
$$

For the former case, $\tilde{d}$ is updated to $-\tilde{d}$ and for the latter case, $\tilde{d}$ remains unchanged.

\subsection{Final update of the multiplicative factor}

5.3.1. Case that $\operatorname{deg}\left(f_{i}\right)=6$. In this case, $\tilde{d}$ is updated to $\tilde{d} \cdot \operatorname{det}\left(M_{i}\right)^{-1}$, where

$$
M_{i}:=\left(\begin{array}{lll}
a_{0} a_{1} & -\left(a_{0}+a_{1}\right) & 1 \\
a_{2} a_{3} & -\left(a_{2}+a_{3}\right) & 1 \\
a_{4} a_{5} & -\left(a_{4}+a_{5}\right) & 1
\end{array}\right) .
$$

Then, $\operatorname{det}\left(M_{i}\right)$ is equal to the determinant of the following $2 \times 2$ matrix:

$$
M_{i}^{0}:=\left(\begin{array}{cc}
\lambda_{1}-\lambda_{0} & \vartheta_{2} \\
\lambda_{2}-\lambda_{0} & -\vartheta_{1}
\end{array}\right) .
$$

We see that $\vartheta_{1}+\vartheta_{2}=-\vartheta_{0}$ by direct calculation. Hence, $\operatorname{det}\left(M_{i}^{0}\right)=-\sum_{j=1}^{2} \vartheta_{j}$ $\left(\lambda_{j}-\lambda_{0}\right)=-\sum_{j=1}^{2} \vartheta_{j} \lambda_{j}+\left(\vartheta_{1}+\vartheta_{2}\right) \lambda_{0}=-\sum_{j=1}^{2} \vartheta_{j} \lambda_{j}-\vartheta_{0} \lambda_{0}=-\sum_{j=0}^{2} \lambda_{j} \vartheta_{j}$. That is,

$$
\operatorname{det}\left(M_{i}\right)=-\sum_{j=0}^{2} \lambda_{j} \vartheta_{j}
$$


5.3.2. Case that $\operatorname{deg}\left(\boldsymbol{f}_{\boldsymbol{i}}\right)=\mathbf{5}$. Assume that $\operatorname{deg}\left(G_{i, j_{0}}\right)=1, a_{m_{0}}$ is the zero of $G_{i, j_{0}}(X)$, and $a_{m_{1}}=\infty$. Let $\lambda_{j_{0}+1}:=a_{2\left(j_{0}+1\right)} a_{2\left(j_{0}+1\right)+1}, \lambda_{j_{0}+2}:=$ $a_{2\left(j_{0}+2\right)} a_{2\left(j_{0}+2\right)+1}$, and $\vartheta_{j_{0}}:=a_{2\left(j_{0}+1\right)}+a_{2\left(j_{0}+1\right)+1}-a_{2\left(j_{0}+2\right)}-a_{2\left(j_{0}+2\right)+1}$. Then, $\tilde{d}$ is updated to $\tilde{d} \cdot \operatorname{det}\left(M_{i}\right)^{-1}$, where $M_{i}$ is constructed from the coefficients of $G_{i, 0}(X), G_{i, 1}(X)$, and $G_{i, 2}(X)$. Here, since $G_{i, j_{0}}(X)$ is linear, the quadratic coefficient of it is 0 . From direct computation,

$$
\operatorname{det}\left(M_{i}\right)=a_{m_{0}} \vartheta_{j_{0}}-\lambda_{j_{0}+1}+\lambda_{j_{0}+2} \text {. }
$$

Then, if we let $\lambda_{j_{0}}:=-a_{m_{0}}, \vartheta_{j_{0}+1}:=1$ and $\vartheta_{j_{0}+2}:=-1$, we see that $(17)$ holds similar to the case that $\operatorname{deg}\left(f_{i}\right)=6$.

\subsection{Description of the algorithm}

Algorithm 1 gives the computation of a Richelot isogeny sequence, and Algorithm 2 gives the computation of a Richelot isogeny. Algorithm 2 computes $\widetilde{S}_{i+1}$ from $S_{i}$ according to the explicit formulas for $\tilde{a}_{0}, \ldots, \tilde{a}_{5}$ and $\tilde{d}$ given in Sections 5.2 and 5.3.

Algorithm 1 iterates Algorithm $2 n$-times, and uses the Perm function to choose the next edge according to $\omega$. Step 3 of Algorithm 1 checks whether $\tilde{d}_{i}=\perp$ or not, and if so, then Algorithm 1 also returns $\perp$ (See Section 3.3). We observed that such split cases rarely occur when starting at $J_{C^{I}}$ or $J_{C^{I I}}$ and $p \geq 2^{160}$.

We fix a branch of square roots, $\delta^{\frac{1}{2}}$, in Algorithm 2 as follows: Fix $\tau \in \mathbb{F}_{q}$

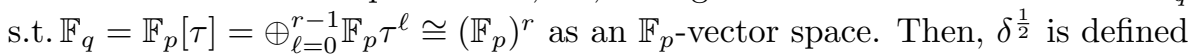
as the max of the two branches using a natural lexicographic order of $\mathbb{F}_{q} \cong$ $\left(\mathbb{F}_{p}\right)^{r}$.

\subsection{Cost of a Richelot operator computation}

We give the cost of the computation of one Richelot isogeny, and we explain the cost of the dominant case that all $G_{j}(X)$ and $\tilde{G}_{j}(X)$ are quadratics. Then, we see that from Algorithm 2, the total cost is 25 multiplications, 4 inversions, and 3 square root computations in $\mathbb{F}_{q}$.

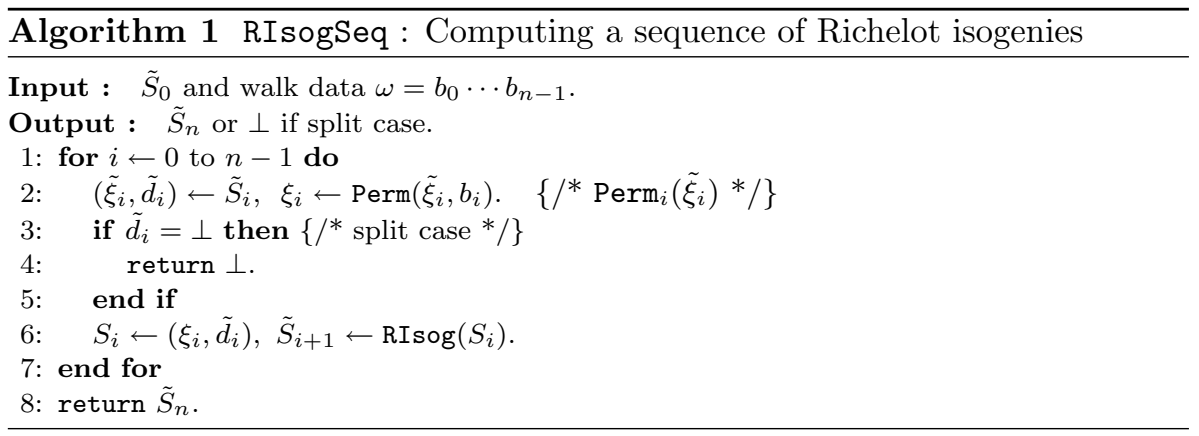




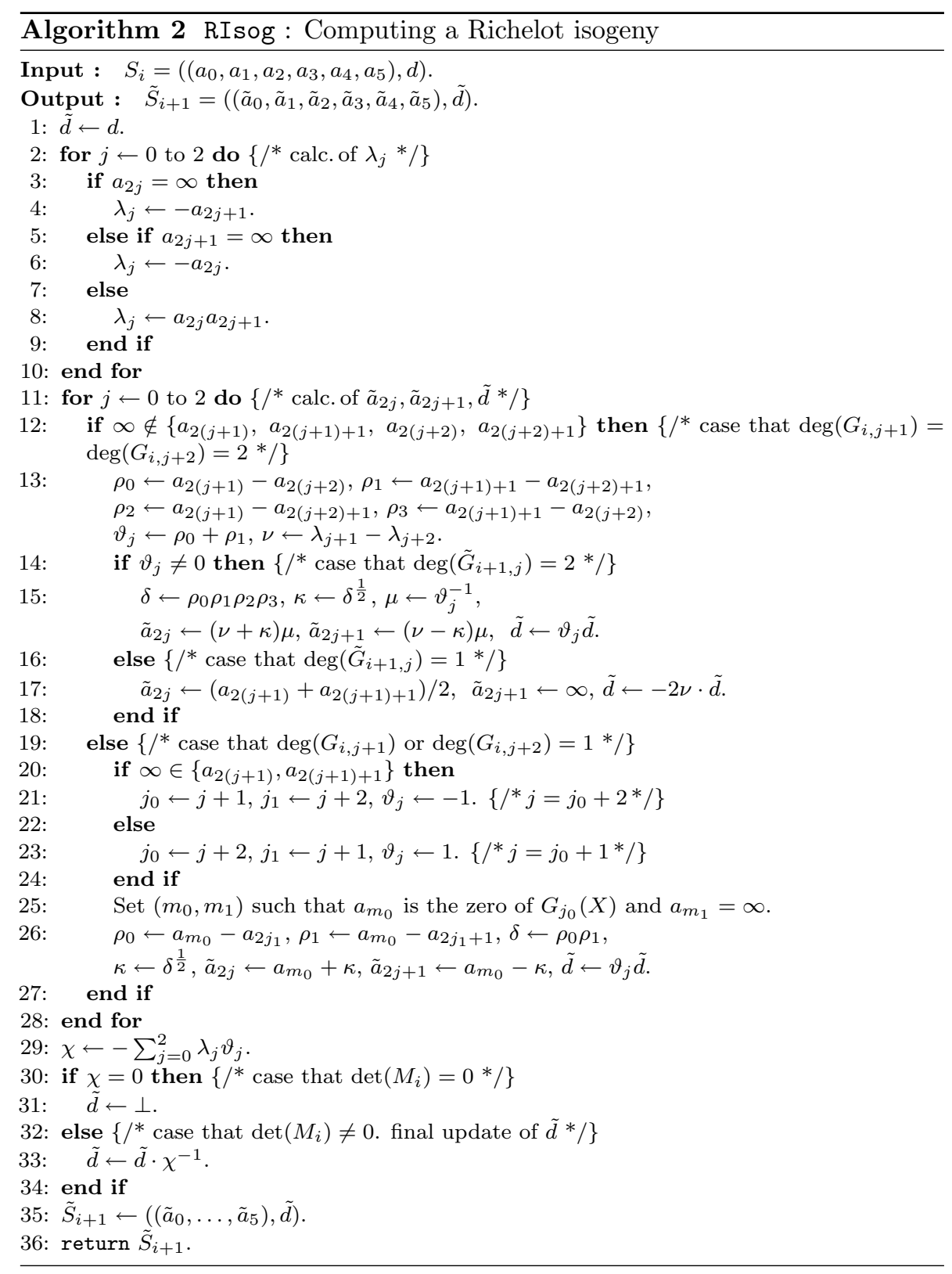

\section{References}

[1] P. R. Bending, Curves of genus 2 with $\sqrt{2}$ multiplication, Ph. D. Thesis, University of Oxford, 1998. 
[2] J.-B. Bost and J.-F. Mestre, Moyenne arithmético-géométrique et périodes des courbes de genre 1 et 2, Gaz. Math. No. 38 (1988), 36-64.

[3] J. W. S. Cassels and E. V. Flynn, Prolegomena to a Middlebrow Arithmetic of Curves of Genus 2, London Mathematical Society Lecture Note Series, 230. Cambridge University Press, Cambridge, 1996.

[4] H. Cohen and G. Frey et al., Handbook of Elliptic and Hyperelliptic Curve Cryptography, Chapman \& Hall, 2006.

[5] D. X. Charles, E. Z. Goren, and K. E. Lauter, Cryptographic hash functions from expander graphs, to appear in Journal of Cryptology.

[6] - Families of Ramanujan graphs and quaternion algebras, to appear in AMSCRM volume "Groups and Symmetries" in honor of John McKay.

[7] Y. J. Choie, E. K. Jeong, and E. J. Lee, Supersingular hyperelliptic curves of genus 2 over finite fields, Appl. Math. Comput. 163 (2005), no. 2, 565-576.

[8] T. Ibukiyama, T. Katsura, and F. Oort, Supersingular curves of genus two and class numbers, Compositio Math. 57 (1986), no. 2, 127-152.

[9] S. Paulus and H.-G. Rück, Real and imaginary quadratic representations of hyperelliptic function fields, Math. Comp. 68 (1999), no. 227, 1233-1241.

[10] S. Paulus and A. Stein, Comparing real and imaginary arithmetics for divisor class groups of hyperelliptic curves, Algorithmic number theory (Portland, OR, 1998), 576591, Lecture Notes in Comput. Sci., 1423, Springer, Berlin, 1998.

[11] A. Rostovtsev and A. Stolbunov, Public-key cryptosystem based on isogenies, preprint, IACR ePrint 2006/145.

[12] B. Smith, Explicit endomorphisms and correspondences, Ph. D. Thesis, The Univ. of Sydney, 2005.

[13] J. Tate, Endomorphisms of abelian varieties over finite fields, Invent. Math. 2 (1966), 134-144.

[14] J. Vélu, Isogénies entre courbes elliptiques, C. R. Acad. Sci. Paris Sér. A-B 273 (1971), A238-A241.

[15] C. Xing, On supersingular abelian varieties of dimension two over finite fields, Finite Fields Appl. 2 (1996), no. 4, 407-421.

[16] R. Yoshida and K. Takashima, Simple algorithms for computing a sequence of 2isogenies, ICISC 2008, LNCS No. 5461, pp. 52-65, Springer Verlag, 2009.

KATSUYUKI TAKASHIMA

Information TeChnology R\&D Center

Mitsubishi Electric

KAMAKURA-SHI, KANAGAWA 247-8501, JAPAN

E-mail address: Takashima.Katsuyuki@aj.MitsubishiElectric.co.jp

REO YOSHIDA

DEPARTMENT OF SOCIAL INFORMATICS

Graduate School of Informatics

KYOTO UNIVERSITY

SAKYO-KU, KYOTO 606-8501, JAPAN

E-mail address: yoshida@ai.soc.i.kyoto-u.ac.jp 